\title{
Scaling of energy spectra in weakly compressible turbulence
}

\author{
Yufeng Dong1 ${ }^{1}$ Guowei He ${ }^{1,2}$
}

Received: 15 February 2017 / Revised: 20 March 2017 / Accepted: 20 March 2017 / Published online: 2 May 2017

(C) The Chinese Society of Theoretical and Applied Mechanics; Institute of Mechanics, Chinese Academy of Sciences and Springer-Verlag Berlin Heidelberg 2017

\begin{abstract}
We find an asymptotic expression for the characteristic timescales of decorrelation processes in weakly compressible and isothermal turbulence. This result is used in the Eddy-Damped Quasi-Normal Markovian equation to derive the scalings of compressible energy spectra: (1) if the acoustic waves are dominant, the compressible energy spectra exhibit $-7 / 3$ scaling; (2) if local eddy straining is dominant, the compressible energy spectra are scaled as -3 . Meanwhile, the energy spectra of incompressible components display the same scaling of $-5 / 3$ as those in incompressible turbulence. The direct numerical simulations of weakly compressible turbulence are used to examine the scaling.
\end{abstract}

Keywords Compressible turbulence - Energy spectra . Two-point closure $\cdot$ Space-time correlation

\section{Introduction}

The universal scaling of energy spectra of velocity fluctuations is fundamentally important to understand turbulent flows. For incompressible turbulence, the universal scaling $-5 / 3$ of energy spectra was originally proposed by Kolmogorov, based on dimensional analysis [1]. This empirical

Guowei He

hgw@lnm.imech.ac.cn

1 The State Key Laboratory of Nonlinear Mechanics, Institute of Mechanics, Chinese Academy of Sciences, No. 15 Beisihuan Xi Road, Beijing 100190, China

2 School of Engineering Sciences, University of Chinese Academy of Sciences, Beijing 100049, China result is further derived from the Navier-Stokes equations by using the two-point closure approaches [2], which leads to the substantial understanding of energy cascade in turbulent flows. However, for compressible turbulence, the dimensional analysis is difficult to be conducted due to nonlinear coupling of velocity, density, and pressure. It remains a great challenge to formulate the universal scaling of compressible energy spectra.

In this paper, we will use a two-point closure approach to derive the universal scaling of energy spectra for compressible and isothermal turbulence. Investigations of the energy spectra in compressible turbulence started with the Burgers equation. An analogy to the Burgers equation [1,3] yields the scaling exponent -2 for compressible velocity spectra. The analogy implies that compressible turbulence is mainly determined by shock waves. Recently, an exact flux relation for compressible turbulence has been formulated to find the scaling exponent $-5 / 3$ for density-weighted velocity spectra [4]. This flux relation reveals the invariant energy flux in the energy cascade of compressible and isothermal turbulence $[4,5]$. The direct kinetic energy cascade for compressible turbulence is proposed through an analytic scaling relation $[6,7]$ and further shown by using the coarse-graining approach [8]. Work by Bertoglio et al. [9-11] uses the direct interaction approximation (DIA) approach to study compressible and isotropic turbulence: they derive the governing equations for energy spectra with undetermined timescales. The characteristic timescales are further taken as the straining timescales of local eddies, which leads to the -3 scaling of compressible energy spectra. This result is crucially dependent on choice of the characteristic timescales.

Characteristic timescales in turbulent flows are essential to the two-point closure approaches [12]. In the Eddy-Damped Quasi-Normal Markovian (EDQNM) approach [13], the 
decorrelation time scales are introduced for eddy damping rates to account for the history-dependence effects. For incompressible turbulence, the decorrelation timescales are taken as the straining time of local eddies [14,15], which leads to Kolmogorov's $-5 / 3$ scaling. However, if the decorrelation timescales are taken as the sweeping time of energy-contained eddies, it yields an incorrect prediction of scaling $-3 / 2$ [16]. For compressible turbulence, dilatational components display the different decorrelation timescales from solenoidal ones $[2,17]$. In this paper, we will find the decorrelation timescales from the space-time correlation models and use them to derive the scalings of compressible energy spectra. The scalings obtained are justified by using their relation with the dominant decorrelation processes in weakly compressible turbulence.

This paper is organized as follows: we will first present the EDQNM equations for both incompressible and compressible components by using the Helmholtz decomposition. Secondly, an asymptotic expression for the decorrelation timescales will be formulated from the swept-wave models. The timescales obtained will be used in the EDQNM equations to find the scaling forms of energy spectra afterward. Finally, we will use the direct numerical simulation (DNS) to investigate the energy spectra obtained for compressible turbulence.

\section{Possible scaling of compressible energy spectra}

We start with the Navier-Stokes equations for weakly compressible and isotropic turbulence. In this case, the density fluctuations are assumed to be much smaller in comparison with their means. In order to isolate the effects of compressibility, it is further assumed that the fluids are barotropic and the speed of sound is constant. These assumptions lead to relate pressure directly with density and circumvent the energy equation, which can be also achieved by assuming the isothermal flows and ideal gas. The Navier-Stokes equations obtained under those assumptions are often used to investigate the compressibility effects and serve as the first step to study compressible turbulence [11].

To study the effects of compressibility to energy spectra, the Helmholtz decomposition [2] can be introduced for velocity fields

$\boldsymbol{u}=\boldsymbol{u}^{\mathrm{s}}+\boldsymbol{u}^{\mathrm{d}}$,

where $\boldsymbol{u}^{\mathrm{s}}$ is a solenoidal component and $\boldsymbol{u}^{\mathrm{d}}$ are a purely compressible or dilatational component. In Fourier space, $\boldsymbol{u}^{\mathrm{s}}$ and $\boldsymbol{u}^{\mathrm{d}}$ can be obtained by the projectors $P_{i j}(\boldsymbol{k}) \equiv \delta_{i j}-k_{i} k_{j} / k^{2}$ and $\Pi_{i j}(\boldsymbol{k}) \equiv k_{i} k_{j} / k^{2}: u_{i}^{\mathrm{s}} \equiv P_{i j}(\boldsymbol{k}) u_{j}(\boldsymbol{k}, t)$ and $u_{i}^{\mathrm{d}} \equiv$ $\Pi_{i j}(\boldsymbol{k}) u_{j}(\boldsymbol{k}, t), i, j=1,2, \ldots$ The governing equations for the solenoidal and dilatational components in Fourier space can be derived from the Navier-Stokes equations. They are given as follows

$$
\begin{aligned}
& \left(\frac{\partial}{\partial t}+\nu k^{2}\right) u_{i}^{\mathrm{s}}(\boldsymbol{k}, t)=-\mathrm{i} \sum_{\boldsymbol{p}+\boldsymbol{q}=\boldsymbol{k}} P_{i j}(\boldsymbol{k}) q_{l} u_{l}(\boldsymbol{p}, t) u_{j}(\boldsymbol{q}, t) \\
& \left(\frac{\partial}{\partial t}+v^{\prime} k^{2}\right) u_{i}^{\mathrm{d}}(\boldsymbol{k}, t)=-\mathrm{i} \sum_{\boldsymbol{p}+\boldsymbol{q}=\boldsymbol{k}} \Pi_{i j}(\boldsymbol{k}) q_{l} u_{l}(\boldsymbol{p}, t) u_{j}(\boldsymbol{q}, t) \\
& \quad-\mathrm{i} \frac{k_{i}}{\rho} p(\boldsymbol{k}, t) \\
& \frac{\partial}{\partial t} p(\boldsymbol{k}, t)=-\mathrm{i} \rho c^{2} k_{i} u_{i}^{\mathrm{d}}(\boldsymbol{k}, t) .
\end{aligned}
$$

Here, $p$ is the pressure and $\rho$ the density. $c \equiv \sqrt{\mathrm{d} p / \mathrm{d} \rho}$ is the speed of sound. $v$ is responsible for molecular viscosity and $v^{\prime}$ is responsible for volume viscosity. For simplicity, we will omit the prime of volume viscosity $v^{\prime}$ without confusion. The symbol $\sum_{\boldsymbol{p}+\boldsymbol{q}=\boldsymbol{k}}$ denotes the integral operator $\iint \delta(\boldsymbol{p}+$ $\boldsymbol{q}-\boldsymbol{k}) \mathrm{d} \boldsymbol{p} \mathrm{d} \boldsymbol{q}$. As expected, the nonlinear terms in Eqs. (2) and (3) are non-local. The new challenges different from incompressible turbulence lie in the coupling of solenoidal, dilatational and pressure components.

Now we apply the EDQNM formalisms to derive the governing equation for solenoidal and dilatational energy spectra, $E^{s s}(k, t) \equiv 2 \pi k^{2}\left\langle u_{i}^{\mathrm{s}}(\boldsymbol{k}, t) u_{i}^{\mathrm{s}}(-\boldsymbol{k}, t)\right\rangle$ and $E^{d d}(k, t) \equiv$ $2 \pi k^{2}\left\langle u_{i}^{\mathrm{d}}(\boldsymbol{k}, t) u_{i}^{\mathrm{d}}(-\boldsymbol{k}, t)\right\rangle$, respectively. The EDQNM procedure for compressible turbulence is similar to the one for incompressible turbulence: the fourth-order moments in the third-order moment equations are approximated by using quasi-normality and eddy damping rates. The third-order moments obtained are then used to represent the energy transfer terms. Finally, a Markovian modification is introduced to simplify the energy transfer terms. A distinct feature for compressible turbulence is that solenoidal and dilatational components are coupled with each other. Under the assumption of weak compressibility, we only retain the linear terms with $E^{d d}$ and discard all terms of high-order $o\left(\left\langle E^{d d}\right\rangle\right)$. Another feature is how to handle the pressure terms. The effects of velocity-velocity-pressure correlations in the third-order moment equations on energy spectra can be represented by an eddy damping. The pressure terms only affect the Green functions and thus change the eddy damping. Therefore, it is reasonable to temporarily remove the pressure terms from the third-order moment equations and recover their effects through the reformulation of eddy damping.

Those considerations yield the following evolutional equations for solenoidal and dilatational energy spectra (seeing "Appendix" section for the details) 


$$
\begin{aligned}
& \left(\frac{\partial}{\partial t}+2 \nu k^{2}\right) E^{s s}(k, t) \\
& =\iint_{\Delta} \theta^{s s}(k, p, q) T(k, p, q, t) \mathrm{d} p \mathrm{~d} q, \\
& \left(\frac{\partial}{\partial t}+2 \nu k^{2}\right) E^{d d}(k, t) \\
& =\iint_{\Delta}\left[\theta^{d d}(k, p, q) \frac{k^{3}}{p q}(x+y z)^{2}\right. \\
& \left.\quad \times E^{s s}(p, t) E^{s s}(q, t)\right] \mathrm{d} p \mathrm{~d} q,
\end{aligned}
$$

where $T(k, p, q, t)$ represents the energy transfer among solenoidal modes $(\boldsymbol{k}, \boldsymbol{p}, \boldsymbol{q}), x, y$, and $z$ are the geometrical coefficients from the triadic interactions of resonant modes $[2,11,13,14]$. The parameters $\theta^{s s}$ and $\theta^{d d}$ are the decorrelation timescales of solenoidal and dilatational components, respectively.

It should be pointed out that only the lowest-order terms for $E^{d d}$ are retained in the present derivations, since $E^{d d}$ is small in the case of weak compressibility. The resulting set of EDQNM equations is consistent with the previous one obtained from DIA [11], where the response functions are used. The energy spectra Eq. (5) for solenoidal components is the same as the one for incompressible turbulence, with the same energy transfer terms $T(k, p, q, t)$. However, the energy spectra Eq. (6) for dilatational components behaves like a forced oscillator [11], where the dilatational modes are driven by the solenoidal modes. This implies that, up to the first-order approximation, the energy cascade approximately holds for solenoidal components. Therefore, the parameter $\theta^{s s}(k, p, q)$ in Eq. (5) play a role of the decorrelation timescales of solenoidal components. It can be thus taken as the straining time of local eddies (or timescales of Lagrangian time correlations [18-20]) $\epsilon^{1 / 3} k^{2 / 3}$, which leads to $E^{s s}(k) \propto k^{-5 / 3}$.

The parameter $\theta^{d d}(k, p, q)$ in Eq. (6) is the characteristic timescales of dilatational components, which represents the history dependence of dilatational components on turbulent flows. According to the DIA and EDQNM theories [14], this timescale is given by

$\theta^{d d}(k, p, q)=\int_{-\infty}^{\infty} G^{d d}(k, t) R^{d d}(p, t) R^{d d}(q, t) \mathrm{d} t$

where $G^{d d}(k, t)$ is the response function and $R^{d d}(p, t)$ the space-time correlation for dilatational components, respectively. The recently developed swept-wave model [17] proposes the space-time correlations for dilatational modes as

$R^{d d}(k, \tau)=\cos (k c \tau) \exp \left(-\mu_{d}^{2} \tau^{2}\right)$
Here, $\mu_{d} \propto \epsilon^{1 / 3} k^{2 / 3}$ are the local straining timescales for Lagrangian time correlations, which replace the sweeping timescales $\mu_{d} \propto V k$ for Eulerian time correlations. The response functions can be re-constructed by including the viscous effect into the space-time correlations. The viscous effect is negligibly small for space-time correlations. However, it is necessarily included into linear response equations. This consideration is consistent with the previous DIA formalism [15] and thus results in

$G^{d d}(k, \tau)=\cos (k c \tau) \exp \left(-\mu_{d}^{2} \tau^{2}\right) \exp \left(-v k^{2} \tau\right)$

Obviously, the first factor $\cos (k c \tau)$ represents the acousticwave effect, the second factor $\exp \left(-\mu_{d}^{2} \tau^{2}\right)$ represents the straining effect of local eddies and the third one $\exp \left(-v k^{2} \tau\right)$, as mentioned above, represents the viscous effect.

For small Mach numbers $M_{t}$, the asymptotic expression for the characteristic timescale can be expanded in the power series of $(k c)^{-1}$ :

$\theta^{d d}=\frac{1}{4} \sum_{\bar{k}} \nu k^{2}\left[\frac{1}{(k c)^{2}}+\frac{2 \alpha^{2}}{(k c)^{4}}+o\left((k c)^{-4}\right)\right]$.

Here, $\bar{k}$ is taken over all combinations of $k \pm p \pm q$ and $\alpha^{2}=\mu_{d}^{2}(k)+\mu_{d}^{2}(p)+\mu_{d}^{2}(q)$. The first term in the sum is of order $(k c)^{-2}$ and the second one in the sum is of order $(k c)^{-4}$. There exist two cases which will be discussed below

(1) If the leading term in Eq. (10) is taken as an approximation to $\theta^{d d}(k, p, q)$, which leads to

$\theta^{d d}(k, p, q) \sim \frac{v k^{2}}{(k c)^{2}}$

Therefore, the submission of the timescale into the EDQNM Eq. (6) leads the power-law solution

$E^{d d}(k) \propto M_{t}^{2} R e^{0} \epsilon^{4 / 3} k^{-7 / 3}$.

This result suggests that the energy spectrum of dilatational components $E^{d d}(k)$ has a scaling of $-7 / 3$. It is also observed that the energy spectrum $E^{d d}(k)$ is dependent on the Mach numbers, but independent of the Reynolds numbers, which are consistent with the properties of energy spectrum in the inertial ranges.

(2) If the second term in Eq. (10) is taken as an approximation to the timescale, $\mu_{d}=\epsilon^{1 / 3} k^{2 / 3}$, the energy spectra are found to be of the power-law form

$E^{d d}(k) \propto M_{t}^{4} R e^{0} \epsilon^{2} k^{-3}$.

This is exactly same as the one obtained by Bertoglio et al. [9-11]. 
It is noted that the timescale $(k c)^{-1}$ is associated with acoustic waves and the timescale $\epsilon^{1 / 3} k^{2 / 3}$ associated with eddy straining. Hence, the above discussions imply that: (1) if acoustic waves are dominating, the dilatational energy spectra exhibit the scaling $-7 / 3$; (2) if eddy straining is dominating, the dilatational energy spectra exhibit the scaling -3 . The decorrelation time scales Eq. (10) show that the time scales associated with acoustic waves are a leading term and the ones associated with eddy straining are a sub-leading term. This implies that the dilatational energy spectra should be scaled as $-7 / 3$.

Furthermore, we calculate the dissipation ratios of dilatational components $\epsilon^{d d}$ to solenoidal one $\epsilon^{s s}$. For the first case, the dissipation ratio is $\epsilon^{d d} / \epsilon^{s s} \propto M_{t}^{2} R e^{-1 / 2}$. This is in agreement with the asymptotic analysis and numerical results by Sarkar et al. [21]. However, for the second case, the dissipation ratio is $\epsilon^{d d} / \epsilon^{s s} \propto M_{t}^{4} R e^{-1} \ln \operatorname{Re}$ [11], which remains controversial in the recent literatures.

\section{Numerical results for compressible energy spectra}

In order to evaluate the possible scaling of energy spectra, we perform DNS $[17,22]$ of compressible and isotropic turbulence with periodic boundary conditions, where an optimized sixth-order compact method and a fourth-order two-step low dissipation and dispersion Runge-Kutta scheme are used to discretize the Navier-Stokes equations. An external force and a uniform cooling are imposed to maintain statistically stationary states. The grid resolution is 256 with the Reynolds number 78 and the Mach number 0.38.

Figure 1 shows the energy co-spectra for solenoidal and dilatational components, respectively. The scaling ranges for co-spectra $k^{5 / 3} E(k)$ and $k^{7 / 3} E(k)$ are visually observable although they are very limited. However, it is also observed that there is no scaling range for the co-spectrum $k^{3} E(k)$. We further calculate the scaling exponents of solenoidal and dilatational energy spectra (not shown here) by using linear regression. These calculations provide the scaling exponent -2.224 for dilatational components and -1.654 for solenoidal component. Therefore, it seems that the present DNS data is in favor of the scaling $-7 / 3$ for dilatational components, although the results suffer from the limited scaling ranges due to low Reynolds numbers. It is noted that previous DNS results present the different scaling exponents for energy spectra at the Mach numbers larger than unity, while the present results are devoted to smaller Mach numbers for weak compressibility. It should be cautious that the definitions of compressible energy spectra are different in the literature. For example, Porter et al. [23] find the scaling $-5 / 3$ for total velocity spectra at unity Mach number; Kritsuk et al. [24] find the scaling $-1.52 \pm 0.01$ for the energy

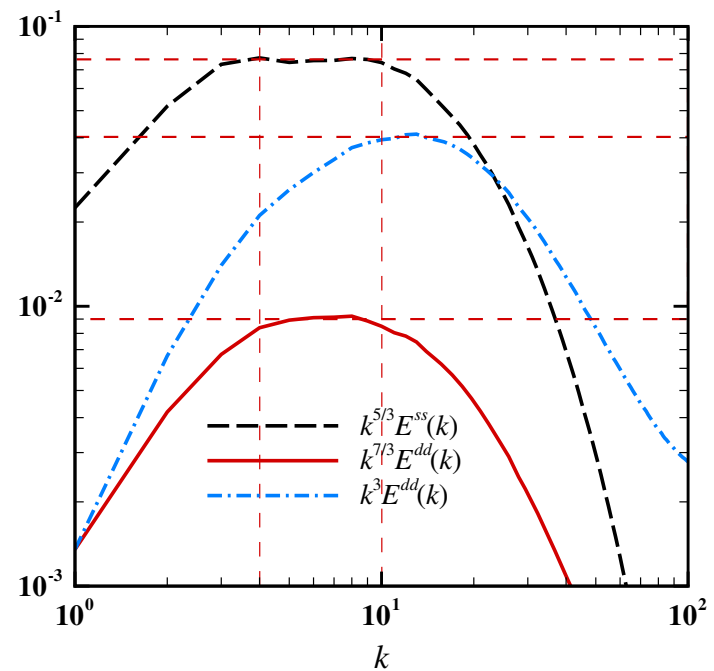

Fig. 1 Co-spectra for kinetic energy of solenoidal and dilatational components from DNS

spectra of density-weighted velocities at high Mach numbers. Work by Wang et al. [25] proposes the scaling -2 for dilatational part and $-5 / 3$ for solenoidal one at the Mach number of one.

\section{Conclusions and discussions}

Bachelor points out characteristic timescales are critically important to a closed set of stochastic models for the NavierStokes equations [26]. In incompressible turbulence, the Lagrangian decorrelation timescales are introduced to the EDQNM models in agreement with the classic Kolmogorov theory. However, in compressible turbulence, the distinct timescales for dilatational components have to be found, although the Lagrangian decorrelation timescales are still valid for solenoidal components. The present work develops a framework to study the possible scaling of compressible energy spectra and their relevant physical processes in terms of space-time correlations.

The weakly compressible and isothermal turbulence can be served as a start point to study compressible turbulence $[27,28]$. At one extreme case of high Mach numbers, the Burgers equation is the analogy of supersonic turbulence, where shock waves are dominate and yield the scaling exponent -2. At another extreme case of low Mach numbers (the weakly compressible and isothermal turbulence), compressible components with a small amount of energy are introduced to interact with incompressible components, where acoustic waves are dominate and yield to scaling of $-7 / 3$. Work by Bertogolio et al. [11] also proposes the alternative that the eddy straining is dominate and the corresponding scaling exponent is -2 . Therefore, it is reasonable 
to consider further the interaction of acoustic waves, shock waves, and eddy straining for compressible energy spectra at moderate Mach numbers.

In summary, we develop an EDQNM model for weakly compressible turbulence. The new development in the EDQNM model is that the eddy-damping rate is determined by the recently developed space-time correlation model, which suggests possible scaling of compressible energy spectra. The leading term in the eddy-damping rate leads to the $-7 / 3$ scaling for dilatational energy spectra, while the subleading term in the eddy damping leads to the -3 scaling. The former implies that dilatational components are dominated by acoustic-wave time scales and the latter implies that dilatational components dominated by straining time scales. Our DNS results cannot exclude the possibility of the $-7 / 3$ scaling. Further work is needed to clarifies the possible scaling of compressible energy spectra in terms of space-time correlations.

Acknowledgements This work was supported by the National Natural Science Foundation of China (Grants 11302238, 11232011, 11572331, and 11490551). The authors would like to acknowledge the support from the Strategic Priority Research Program (Grant XDB22040104) and the Key Research Program of Frontier Sciences of Chinese Academy of Sciences and the National Basic Research Program of China (973 Program) (Grant 2013CB834100: Nonlinear science).

\section{Appendix: The EDQNM equations for weakly compressible turbulence}

We use Eqs. (2)-(4) to find the governing equations for energy spectra of solenoidal and dilatational components, pressure-velocity cross correlations and pressure spectrum

$$
\begin{aligned}
& \left(\frac{\partial}{\partial t}+2 v k^{2}\right)\left\langle u_{i}^{\mathrm{s}}(\boldsymbol{k}, t) u_{i}^{\mathrm{s}}(-\boldsymbol{k}, t)\right\rangle \\
& =-\mathrm{i} \int_{\boldsymbol{p}+\boldsymbol{q}=\boldsymbol{k}} P_{i j}(\boldsymbol{k}) q_{l}\left\langle u_{l}(\boldsymbol{p}, t) u_{j}(\boldsymbol{q}, t) u_{i}^{\mathrm{s}}(-\boldsymbol{k}, t)\right\rangle \mathrm{d} \boldsymbol{p} \mathrm{d} \boldsymbol{q} \\
& \quad-\mathrm{i} \int_{\boldsymbol{p}+\boldsymbol{q}=-\boldsymbol{k}} P_{i j}(-\boldsymbol{k}) q_{l}\left\langle u_{l}(\boldsymbol{p}, t) u_{j}(\boldsymbol{q}, t) u_{i}^{\mathrm{s}}(\boldsymbol{k}, t)\right\rangle \mathrm{d} \boldsymbol{p} \mathrm{d} \boldsymbol{q},
\end{aligned}
$$

$$
\begin{aligned}
& \left(\frac{\partial}{\partial t}+v k^{2}\right)\left\langle u_{i}^{\mathrm{d}}(\boldsymbol{k}, t) u_{i}^{\mathrm{d}}(-\boldsymbol{k}, t)\right\rangle \\
& =-\mathrm{i} \int_{\boldsymbol{p}+\boldsymbol{q}=\boldsymbol{k}} \Pi_{i j}(\boldsymbol{k}) q_{l}\left\langle u_{l}(\boldsymbol{p}, t) u_{j}(\boldsymbol{q}, t) u_{i}^{\mathrm{d}}(-\boldsymbol{k}, t)\right\rangle \mathrm{d} \boldsymbol{p} \mathrm{d} \boldsymbol{q} \\
& \quad-\mathrm{i} \int_{\boldsymbol{p}+\boldsymbol{q}=-\boldsymbol{k}} \Pi_{i j}(-\boldsymbol{k}) q_{l}\left\langle u_{l}(\boldsymbol{p}, t) u_{j}(\boldsymbol{q}, t) u_{i}^{\mathrm{d}}(\boldsymbol{k}, t)\right\rangle \mathrm{d} \boldsymbol{p} \mathrm{d} \boldsymbol{q} \\
& \quad-\mathrm{i} \frac{k_{i}}{\rho}\left\langle p(\boldsymbol{k}, t) u_{i}^{\mathrm{d}}(-\boldsymbol{k}, t)\right\rangle+\mathrm{i} \frac{k_{i}}{\rho}\left\langle p(-\boldsymbol{k}, t) u_{i}^{\mathrm{d}}(\boldsymbol{k}, t)\right\rangle,
\end{aligned}
$$

$$
\begin{aligned}
& \left(\frac{\partial}{\partial t}+v k^{2}\right)\left\langle p(\boldsymbol{k}, t) u_{i}^{\mathrm{d}}(-\boldsymbol{k}, t)\right\rangle \\
& =-\mathrm{i} \int_{\boldsymbol{p}+\boldsymbol{q}=-\boldsymbol{k}} \Pi_{i j}(-\boldsymbol{k}) q_{l}\left\langle p(\boldsymbol{k}, t) u_{l}(\boldsymbol{p}, t) u_{j}(\boldsymbol{q}, t)\right\rangle \mathrm{d} \boldsymbol{p} \mathrm{d} \boldsymbol{q} \\
& \quad-\mathrm{i} \rho c^{2} k_{j}\left\langle u_{j}^{\mathrm{d}}(\boldsymbol{k}, t) u_{i}^{\mathrm{d}}(-\boldsymbol{k}, t)\right\rangle+\mathrm{i} \frac{k_{i}}{\rho}\langle p(\boldsymbol{k}, t) p(-\boldsymbol{k}, t)\rangle,
\end{aligned}
$$

$$
\begin{aligned}
& \frac{\partial}{\partial t}\langle p(\boldsymbol{k}, t) p(-\boldsymbol{k}, t)\rangle \\
& \quad=-\mathrm{i} \rho c^{2} k_{i}\left\langle u_{i}^{\mathrm{d}}(\boldsymbol{k}, t) p(-\boldsymbol{k}, t)\right\rangle+\mathrm{i} \rho c^{2} k_{i}\left\langle u_{i}^{\mathrm{d}}(\boldsymbol{k}, t) p(\boldsymbol{k}, t)\right\rangle .
\end{aligned}
$$

The governing Eq. (A1) for solenoidal components can be simplified as

$$
\begin{aligned}
& \left(\frac{\partial}{\partial t}+2 v k^{2}\right)\left\langle u_{i}^{\mathrm{s}}(\boldsymbol{k}, t) u_{i}^{\mathrm{s}}(-\boldsymbol{k}, t)\right\rangle \\
& =-\mathrm{i} \int_{\boldsymbol{p}+\boldsymbol{q}=\boldsymbol{k}} P_{i j}(\boldsymbol{k}) q_{l} \\
& \quad \times\left[\begin{array}{l}
\left\langle u_{l}(\boldsymbol{p}, t) u_{j}(\boldsymbol{q}, t) u_{i}^{\mathrm{s}}(-\boldsymbol{k}, t)\right\rangle \\
-\left\langle u_{l}(-\boldsymbol{p}, t) u_{j}(-\boldsymbol{q}, t) u_{i}^{\mathrm{s}}(\boldsymbol{k}, t)\right\rangle
\end{array}\right] \mathrm{d} \boldsymbol{p} \mathrm{d} \boldsymbol{q} \\
& =2 \int_{\boldsymbol{p}+\boldsymbol{q}=\boldsymbol{k}} P_{i j}(\boldsymbol{k}) q_{l} \\
& \quad \times \operatorname{Im}\left[\left\langle u_{l}(\boldsymbol{p}, t) u_{j}(\boldsymbol{q}, t) u_{i}^{\mathrm{s}}(-\boldsymbol{k}, t)\right\rangle\right] \mathrm{d} \boldsymbol{p} \mathrm{d} \boldsymbol{q} .
\end{aligned}
$$

Here, $\operatorname{Im}[\cdot]$ denotes the imaginary part of a complex function. The triple correlations in Eq. (A5) satisfy the following equation

$$
\begin{aligned}
& {\left[\frac{\partial}{\partial t}+v\left(k^{2}+p^{2}+q^{2}\right)\right]\left\langle u_{i}^{\mathrm{s}}(-\boldsymbol{k}, t) u_{l}(\boldsymbol{p}, t) u_{j}(\boldsymbol{q}, t)\right\rangle} \\
& =-\mathrm{i} \int_{\boldsymbol{r}+\boldsymbol{s}=-\boldsymbol{k}} P_{i m}(-\boldsymbol{k}) s_{n} \\
& \quad \times\left\langle u_{n}(\boldsymbol{r}, t) u_{m}(\boldsymbol{s}, t) u_{l}(\boldsymbol{p}, t) u_{j}(\boldsymbol{q}, t)\right\rangle \mathrm{d} \boldsymbol{r} \mathrm{d} \boldsymbol{s} \\
& \quad-\mathrm{i} \int_{\boldsymbol{r}+\boldsymbol{s}=\boldsymbol{p}} s_{n}\left\langle u_{n}(\boldsymbol{r}, t) u_{l}(\boldsymbol{s}, t) u_{i}^{\mathrm{s}}(\boldsymbol{k}, t) u_{j}(\boldsymbol{q}, t)\right\rangle \mathrm{d} \boldsymbol{r} \mathrm{d} \boldsymbol{s} \\
& -\mathrm{i} \frac{p_{l}}{\rho}\left\langle u_{i}^{\mathrm{s}}(\boldsymbol{k}, t) p(\boldsymbol{p}, t) u_{j}(\boldsymbol{q}, t)\right\rangle \\
& -\mathrm{i} \int_{\boldsymbol{r}+\boldsymbol{s}=\boldsymbol{q}} s_{n}\left\langle u_{n}(\boldsymbol{r}, t) u_{j}(\boldsymbol{s}, t) u_{l}(\boldsymbol{p}, t) u_{i}^{\mathrm{s}}(\boldsymbol{k}, t)\right\rangle \mathrm{d} \boldsymbol{r} \mathrm{d} \boldsymbol{s} \\
& -\mathrm{i} \frac{q_{j}}{\rho}\left\langle u_{i}^{\mathrm{s}}(\boldsymbol{k}, t) u_{l}(\boldsymbol{p}, t) p(\boldsymbol{q}, t)\right\rangle .
\end{aligned}
$$

The quasi-normality assumption is introduced into Eq. (A6), which leads to 


$$
\begin{aligned}
& {\left[\frac{\partial}{\partial t}+v\left(k^{2}+p^{2}+q^{2}\right)\right]\left\langle u_{i}^{\mathrm{s}}(-\boldsymbol{k}, t) u_{l}(\boldsymbol{p}, t) u_{j}(\boldsymbol{q}, t)\right\rangle} \\
& =-\mathrm{i} \int_{\boldsymbol{r}+\boldsymbol{s}=-\boldsymbol{k}} P_{i m}(-\boldsymbol{k}) \\
& \times\left[\begin{array}{l}
s_{n}\left\langle u_{n}(\boldsymbol{r}, t) u_{m}(\boldsymbol{s}, t)\right\rangle\left\langle u_{l}(\boldsymbol{p}, t) u_{j}(\boldsymbol{q}, t)\right\rangle \\
+s_{n}\left\langle u_{n}(\boldsymbol{r}, t) u_{l}(\boldsymbol{p}, t)\right\rangle\left\langle u_{m}(\boldsymbol{s}, t) u_{j}(\boldsymbol{q}, t)\right\rangle \\
+s_{n}\left\langle u_{n}(\boldsymbol{r}, t) u_{j}(\boldsymbol{q}, t)\right\rangle\left\langle u_{m}(\boldsymbol{s}, t) u_{l}(\boldsymbol{p}, t)\right\rangle
\end{array}\right] \mathrm{d} \boldsymbol{r} \mathrm{s} \\
& -\mathrm{i} \int_{\boldsymbol{r}+\boldsymbol{s}=\boldsymbol{p}}\left[\begin{array}{l}
s_{n}\left\langle u_{n}(\boldsymbol{r}, t) u_{l}(\boldsymbol{s}, t)\right\rangle\left\langle u_{i}^{\mathrm{s}}(-\boldsymbol{k}, t) u_{j}(\boldsymbol{q}, t)\right\rangle \\
+s_{n}\left\langle u_{n}(\boldsymbol{r}, t) u_{i}^{\mathrm{s}}(-\boldsymbol{k}, t)\right\rangle\left\langle u_{l}(\boldsymbol{s}, t) u_{j}(\boldsymbol{q}, t)\right\rangle \\
+s_{n}\left\langle u_{n}(\boldsymbol{r}, t) u_{j}(\boldsymbol{q}, t)\right\rangle\left\langle u_{l}(\boldsymbol{s}, t) u_{i}^{\mathrm{s}}(-\boldsymbol{k}, t)\right\rangle
\end{array}\right] \mathrm{d} \boldsymbol{d} \mathrm{d} \boldsymbol{s} \\
& -\mathrm{i} \int_{\boldsymbol{r}+\boldsymbol{s}=\boldsymbol{q}}\left[\begin{array}{l}
s_{n}\left\langle u_{n}(\boldsymbol{r}, t) u_{j}(\boldsymbol{s}, t)\right\rangle\left\langle u_{l}(\boldsymbol{p}, t) u_{i}^{\mathrm{s}}(-\boldsymbol{k}, t)\right\rangle \\
+s_{n}\left\langle u_{n}(\boldsymbol{r}, t) u_{l}(\boldsymbol{p}, t)\right\rangle\left\langle u_{j}(\boldsymbol{s}, t) u_{i}^{\mathrm{s}}(-\boldsymbol{k}, t)\right\rangle \\
+s_{n}\left\langle u_{n}(\boldsymbol{r}, t) u_{i}^{\mathrm{s}}(-\boldsymbol{k}, t)\right\rangle\left\langle u_{j}(\boldsymbol{s}, t) u_{l}(\boldsymbol{p}, t)\right\rangle
\end{array}\right] \mathrm{d} \boldsymbol{d} \mathrm{d} \boldsymbol{s} .
\end{aligned}
$$

For weakly compressible turbulence, where the Mach number $M_{t}$ is very small, the identities $\left\langle u_{i} u_{i}\right\rangle \equiv\left\langle u_{i}^{\mathrm{s}} u_{i}^{\mathrm{s}}\right\rangle+\left\langle u_{i}^{\mathrm{d}} u_{i}^{\mathrm{d}}\right\rangle$ and $\lim _{M_{t} \rightarrow 0}\left\langle u_{i} u_{i}\right\rangle=\left\langle u_{i}^{\mathrm{s}} u_{i}^{\mathrm{s}}\right\rangle$ imply $\lim _{M_{t} \rightarrow 0}\left\langle u_{i}^{\mathrm{d}} u_{i}^{\mathrm{d}}\right\rangle=0$, which leads to $\left\langle u_{i}^{\mathrm{d}} u_{i}^{\mathrm{d}}\right\rangle \ll\left\langle u_{i}^{\mathrm{s}} u_{i}^{\mathrm{s}}\right\rangle$. Therefore, Eq. (A7) can be further simplified as

$$
\begin{aligned}
& {\left[\frac{\partial}{\partial t}+v\left(k^{2}+p^{2}+q^{2}\right)\right]\left\langle u_{i}^{\mathrm{s}}(-\boldsymbol{k}, t) u_{l}(\boldsymbol{p}, t) u_{j}(\boldsymbol{q}, t)\right\rangle} \\
& =\mathrm{i} P_{i m}(\boldsymbol{k})\left[\begin{array}{l}
q_{n}\left\langle u_{n}^{\mathrm{s}}(-\boldsymbol{p}, t) u_{l}^{\mathrm{s}}(\boldsymbol{p}, t)\right\rangle\left\langle u_{m}^{\mathrm{s}}(-\boldsymbol{q}, t) u_{j}^{\mathrm{s}}(\boldsymbol{q}, t)\right\rangle \\
+p_{n}\left\langle u_{n}^{\mathrm{s}}(-\boldsymbol{q}, t) u_{j}^{\mathrm{s}}(\boldsymbol{q}, t)\right\rangle\left\langle u_{m}^{\mathrm{s}}(-\boldsymbol{p}, t) u_{l}^{\mathrm{s}}(\boldsymbol{p}, t)\right\rangle
\end{array}\right] \\
& +\mathrm{i}\left[\begin{array}{l}
q_{n}\left\langle u_{n}^{\mathrm{s}}(\boldsymbol{k}, t) u_{i}^{\mathrm{s}}(-\boldsymbol{k}, t)\right\rangle\left\langle u_{l}^{\mathrm{s}}(-\boldsymbol{q}, t) u_{j}^{\mathrm{s}}(\boldsymbol{q}, t)\right\rangle \\
-k_{n}\left\langle u_{n}^{\mathrm{s}}(-\boldsymbol{q}, t) u_{j}^{\mathrm{s}}(\boldsymbol{q}, t)\right\rangle\left\langle u_{l}^{\mathrm{s}}(\boldsymbol{k}, t) u_{i}^{\mathrm{s}}(-\boldsymbol{k}, t)\right\rangle
\end{array}\right] \\
& +\mathrm{i}\left[\begin{array}{l}
-k_{n}\left\langle u_{n}^{\mathrm{s}}(-\boldsymbol{p}, t) u_{l}^{\mathrm{s}}(\boldsymbol{p}, t)\right\rangle\left\langle u_{j}^{\mathrm{s}}(\boldsymbol{k}, t) u_{i}^{\mathrm{s}}(-\boldsymbol{k}, t)\right\rangle \\
+p_{n}\left\langle u_{n}^{\mathrm{s}}(\boldsymbol{k}, t) u_{i}^{\mathrm{s}}(-\boldsymbol{k}, t)\right\rangle\left\langle u_{j}^{\mathrm{s}}(-\boldsymbol{p}, t) u_{l}^{\mathrm{s}}(\boldsymbol{p}, t)\right\rangle
\end{array}\right] .
\end{aligned}
$$

As a result, we could obtain the triple correlations from Eq. (A8)

$$
\begin{aligned}
& \left\langle u_{l}(\boldsymbol{p}, t) u_{j}(\boldsymbol{q}, t) u_{i}^{\mathrm{s}}(-\boldsymbol{k}, t)\right\rangle \\
& \quad=\mathrm{i} \int_{-\infty}^{t} \mathrm{e}^{-v\left(k^{2}+p^{2}+q^{2}\right)\left(t-t^{\prime}\right)} T_{i l j}\left(\boldsymbol{k}, \boldsymbol{p}, \boldsymbol{q}, t^{\prime}\right) \mathrm{d} t^{\prime},
\end{aligned}
$$

where

$$
\begin{aligned}
& T_{i l j}(\boldsymbol{k}, \boldsymbol{p}, \boldsymbol{q}, t) \\
& =P_{i m}(\boldsymbol{k})\left[\begin{array}{l}
q_{n}\left\langle u_{n}^{\mathrm{s}}(-\boldsymbol{p}, t) u_{l}^{\mathrm{s}}(\boldsymbol{p}, t)\right\rangle\left\langle u_{m}^{\mathrm{s}}(-\boldsymbol{q}, t) u_{j}^{\mathrm{s}}(\boldsymbol{q}, t)\right\rangle \\
+p_{n}\left\langle u_{n}^{\mathrm{s}}(-\boldsymbol{q}, t) u_{j}^{\mathrm{s}}(\boldsymbol{q}, t)\right\rangle\left\langle u_{m}^{\mathrm{s}}(-\boldsymbol{p}, t) u_{l}^{\mathrm{s}}(\boldsymbol{p}, t)\right\rangle
\end{array}\right]
\end{aligned}
$$

$$
\begin{aligned}
& +\left[\begin{array}{c}
q_{n}\left\langle u_{n}^{\mathrm{s}}(\boldsymbol{k}, t) u_{i}^{\mathrm{s}}(-\boldsymbol{k}, t)\right\rangle\left\langle u_{l}^{\mathrm{s}}(-\boldsymbol{q}, t) u_{j}^{\mathrm{s}}(\boldsymbol{q}, t)\right\rangle \\
-k_{n}\left\langle u_{n}^{\mathrm{s}}(-\boldsymbol{q}, t) u_{j}^{\mathrm{s}}(\boldsymbol{q}, t)\right\rangle\left\langle u_{l}^{\mathrm{s}}(\boldsymbol{k}, t) u_{i}^{\mathrm{s}}(-\boldsymbol{k}, t)\right\rangle
\end{array}\right] \\
& +\left[\begin{array}{c}
-k_{n}\left\langle u_{n}^{\mathrm{s}}(-\boldsymbol{p}, t) u_{l}^{\mathrm{s}}(\boldsymbol{p}, t)\right\rangle\left\langle u_{j}^{\mathrm{s}}(\boldsymbol{k}, t) u_{i}^{\mathrm{s}}(-\boldsymbol{k}, t)\right\rangle \\
+p_{n}\left\langle u_{n}^{\mathrm{s}}(\boldsymbol{k}, t) u_{i}^{\mathrm{s}}(-\boldsymbol{k}, t)\right\rangle\left\langle u_{j}^{\mathrm{s}}(-\boldsymbol{p}, t) u_{l}^{\mathrm{s}}(\boldsymbol{p}, t)\right\rangle
\end{array}\right] .
\end{aligned}
$$

Submission of the triple correlations into Eq. (A5) leads to the closed equations for solenoidal energy spectrum

$$
\begin{aligned}
& \left(\frac{\partial}{\partial t}+2 v k^{2}\right)\left\langle u_{i}^{\mathrm{s}}(\boldsymbol{k}, t) u_{i}^{\mathrm{s}}(-\boldsymbol{k}, t)\right\rangle=2 \int_{\boldsymbol{p}+\boldsymbol{q}=\boldsymbol{k}} P_{i j}(\boldsymbol{k}) q_{l} \\
& \quad \times\left[\int_{-\infty}^{t} \mathrm{e}^{-v\left(k^{2}+p^{2}+q^{2}\right)\left(t-t^{\prime}\right)} T_{i l j}\left(\boldsymbol{k}, \boldsymbol{p}, \boldsymbol{q}, t^{\prime}\right) \mathrm{d} t^{\prime}\right] \mathrm{d} \boldsymbol{p} \mathrm{d} \boldsymbol{q} .
\end{aligned}
$$

We further use the Markovian assumption to Eq. (A11), which yields

$$
\begin{aligned}
& \left(\frac{\partial}{\partial t}+2 v k^{2}\right)\left\langle u_{i}^{\mathrm{s}}(\boldsymbol{k}, t) u_{i}^{\mathrm{s}}(-\boldsymbol{k}, t)\right\rangle=2 \int_{\boldsymbol{p}+\boldsymbol{q}=\boldsymbol{k}} P_{i j}(\boldsymbol{k}) q_{l} \\
& \quad \times\left[\int_{-\infty}^{t} \mathrm{e}^{-v\left(k^{2}+p^{2}+q^{2}\right)\left(t-t^{\prime}\right)} \mathrm{d} t^{\prime}\right] T_{i l j}(\boldsymbol{k}, \boldsymbol{p}, \boldsymbol{q}, t) \mathrm{d} \boldsymbol{p} \mathrm{d} \boldsymbol{q} .
\end{aligned}
$$

According to Eq. (A12), we can obtain the governing equations for solenoidal energy spectrum $E^{s s}(k, t) \equiv$ $2 \pi k^{2}\left\langle u_{i}^{\mathrm{s}}(\boldsymbol{k}, t) u_{i}^{\mathrm{s}}(-\boldsymbol{k}, t)\right\rangle$,

$$
\begin{aligned}
& \left(\frac{\partial}{\partial t}+v k^{2}\right) E^{s s}(k, t) \\
& =\int_{\substack{p+q=k\\
}}\left[\int_{-\infty}^{t} \mathrm{e}^{-v\left(k^{2}+p^{2}+q^{2}\right)\left(t-t^{\prime}\right)} \mathrm{d} t^{\prime}\right] \\
& \quad \times T(k, p, q, t) \mathrm{d} p \mathrm{~d} q,
\end{aligned}
$$

$T(k, p, q, t)$

$$
\begin{aligned}
= & \frac{k^{3}}{p q} a(k, p, q) E^{s s}(p, t) E^{s s}(q, t) \\
& -\frac{k}{2 p q} E^{s s}(k, t)\left[p^{2} b(k, p, q) E^{s s}(q, t)\right. \\
& \left.+q^{2} b(k, q, p) E^{s s}(p, t)\right],
\end{aligned}
$$

which are exactly the same as the equations of energy spectrum in incompressible turbulence.

The governing Eq. (A2) for compressible components can be further simplified

$$
\begin{aligned}
& \left(\frac{\partial}{\partial t}+v k^{2}\right)\left\langle u_{i}^{\mathrm{d}}(\boldsymbol{k}, t) u_{i}^{\mathrm{d}}(-\boldsymbol{k}, t)\right\rangle \\
& \quad=-\mathrm{i} \int_{\boldsymbol{p}+\boldsymbol{q}=\boldsymbol{k}} \Pi_{i j}(\boldsymbol{k}) q_{l}\left\langle u_{l}(\boldsymbol{p}, t) u_{j}(\boldsymbol{q}, t) u_{i}^{\mathrm{d}}(-\boldsymbol{k}, t)\right\rangle \mathrm{d} \boldsymbol{p} \mathrm{d} \boldsymbol{q}
\end{aligned}
$$




$$
\begin{aligned}
& -\mathrm{i} \int_{\boldsymbol{p}+\boldsymbol{q}=-\boldsymbol{k}} \Pi_{i j}(-\boldsymbol{k}) q_{l}\left\langle u_{l}(\boldsymbol{p}, t) u_{j}(\boldsymbol{q}, t) u_{i}^{\mathrm{d}}(\boldsymbol{k}, t)\right\rangle \mathrm{d} \boldsymbol{p} \mathrm{d} \boldsymbol{q} \\
= & 2 \int_{\boldsymbol{p}+\boldsymbol{q}=\boldsymbol{k}} \Pi_{i j}(\boldsymbol{k}) q_{l} \\
& \times \operatorname{Im}\left[\left\langle u_{l}(\boldsymbol{p}, t) u_{j}(\boldsymbol{q}, t) u_{i}^{\mathrm{d}}(-\boldsymbol{k}, t)\right\rangle\right] \mathrm{d} \boldsymbol{p} \mathrm{d} \boldsymbol{q} .
\end{aligned}
$$

Here, the statistical stationarity $\frac{\partial}{\partial t}\langle p(\boldsymbol{k}, t) p(-\boldsymbol{k}, t)\rangle=0$ and the governing Eq. (A4) for pressure spectrum are used. As a result, we obtain the identity $\left\langle u_{i}^{\mathrm{d}}(\boldsymbol{k}, t) p(-\boldsymbol{k}, t)\right\rangle \equiv$ $\left\langle u_{i}^{\mathrm{d}}(-\boldsymbol{k}, t) p(\boldsymbol{k}, t)\right\rangle$ and that the sum of terms associated with pressure is zero in Eq. (A2).

Therefore, the triple correlations in Eq. (A15) satisfy the equation below

$$
\begin{aligned}
& {\left[\frac{\partial}{\partial t}+v\left(k^{2}+p^{2}+q^{2}\right)\right]\left\langle u_{i}^{\mathrm{d}}(-\boldsymbol{k}, t) u_{l}(\boldsymbol{p}, t) u_{j}(\boldsymbol{q}, t)\right\rangle} \\
& =-\mathrm{i} \int_{\boldsymbol{r}+\boldsymbol{s}=-\boldsymbol{k}} \Pi_{i m}(-\boldsymbol{k}) s_{n} \\
& \quad \times\left\langle u_{n}(\boldsymbol{r}, t) u_{m}(\boldsymbol{s}, t) u_{l}(\boldsymbol{p}, t) u_{j}(\boldsymbol{q}, t)\right\rangle \mathrm{d} \boldsymbol{r} \mathrm{d} \boldsymbol{s} \\
& \quad+\mathrm{i} \frac{k_{i}}{\rho}\left\langle p(-\boldsymbol{k}, t) u_{l}(\boldsymbol{p}, t) u_{j}(\boldsymbol{q}, t)\right\rangle \\
& \quad-\mathrm{i} \int_{\boldsymbol{r}+\boldsymbol{s}=\boldsymbol{p}} s_{n}\left\langle u_{n}(\boldsymbol{r}, t) u_{l}(\boldsymbol{s}, t) u_{i}^{\mathrm{d}}(-\boldsymbol{k}, t) u_{j}(\boldsymbol{q}, t)\right\rangle \mathrm{d} \boldsymbol{r} \mathrm{d} \boldsymbol{s} \\
& \quad-\mathrm{i} \frac{p_{l}}{\rho}\left\langle u_{i}^{\mathrm{d}}(-\boldsymbol{k}, t) p(\boldsymbol{p}, t) u_{j}(\boldsymbol{q}, t)\right\rangle \\
& \quad-\mathrm{i} \int_{\boldsymbol{r}+\boldsymbol{s}=\boldsymbol{q}} s_{n}\left\langle u_{n}(\boldsymbol{r}, t) u_{j}(\boldsymbol{s}, t) u_{l}(\boldsymbol{p}, t) u_{i}^{\mathrm{d}}(-\boldsymbol{k}, t)\right\rangle \mathrm{d} \boldsymbol{r} \mathrm{d} \boldsymbol{s} \\
& \quad-\mathrm{i} \frac{q_{j}}{\rho}\left\langle u_{i}^{\mathrm{d}}(-\boldsymbol{k}, t) u_{l}(\boldsymbol{p}, t) p(\boldsymbol{q}, t)\right\rangle .
\end{aligned}
$$

In the following, the quasi-normality assumption is introduced to express the quadruple correlations as the products of double correlations; the effects of the velocity-velocitypressure correlations on the energy spectra will be considered as the damping factors; the weakly compressibility assumption is also introduced so that $\left\langle u^{\mathrm{s}} u^{\mathrm{s}}\right\rangle \gg\left\langle u^{\mathrm{d}} u^{\mathrm{d}}\right\rangle$, which implies that the terms associated with $\left\langle u^{\mathrm{d}} u^{\mathrm{d}}\right\rangle$ are negligibly small. As a result, we obtain the triple correlations

$$
\begin{aligned}
& \left\langle u_{i}^{\mathrm{d}}(-\boldsymbol{k}, t) u_{l}(\boldsymbol{p}, t) u_{j}(\boldsymbol{q}, t)\right\rangle=\mathrm{i} \int_{-\infty}^{t} \mathrm{e}^{-v\left(k^{2}+p^{2}+q^{2}\right)\left(t-t^{\prime}\right)} \\
& \times\left[\begin{array}{l}
\Pi_{i m}(\boldsymbol{k}) q_{n}\left\langle u_{n}^{\mathrm{s}}\left(-\boldsymbol{p}, t^{\prime}\right) u_{l}^{\mathrm{s}}\left(\boldsymbol{p}, t^{\prime}\right)\right\rangle\left\langle u_{m}^{\mathrm{s}}\left(-\boldsymbol{q}, t^{\prime}\right) u_{j}^{\mathrm{s}}\left(\boldsymbol{q}, t^{\prime}\right)\right\rangle \\
+\Pi_{i m}(\boldsymbol{k}) p_{n}\left\langle u_{n}^{\mathrm{s}}\left(-\boldsymbol{q}, t^{\prime}\right) u_{j}^{\mathrm{s}}\left(\boldsymbol{q}, t^{\prime}\right)\right\rangle\left\langle u_{m}^{\mathrm{s}}\left(-\boldsymbol{p}, t^{\prime}\right) u_{l}^{\mathrm{s}}\left(\boldsymbol{p}, t^{\prime}\right)\right\rangle
\end{array}\right] \mathrm{d} t^{\prime} .
\end{aligned}
$$

Substituting the triple correlations into Eq. (A15) and noting that the energy spectrum is defined as $E^{d d}(k, t) \equiv$ $2 \pi k^{2}\left\langle u_{i}^{\mathrm{d}}(\boldsymbol{k}, t) u_{i}^{\mathrm{d}}(-\boldsymbol{k}, t)\right\rangle$, we obtain

$$
\begin{aligned}
& \left(\frac{\partial}{\partial t}+v k^{2}\right) E^{d d}(k, t)=\int_{p+q=k} \int_{-\infty}^{t} \mathrm{e}^{-v\left(k^{2}+p^{2}+q^{2}\right)\left(t-t^{\prime}\right)} \\
& \times \frac{k^{3}}{2 p q} f(k, p, q) E^{s s}\left(p, t^{\prime}\right) E^{s s}\left(q, t^{\prime}\right) \mathrm{d} t^{\prime} \mathrm{d} p \mathrm{~d} q,
\end{aligned}
$$

where

$$
f(k, p, q)=\frac{1}{k^{2}}\left[\begin{array}{l}
\Pi_{i j}(\boldsymbol{k}) q_{l} \Pi_{i m}(\boldsymbol{k}) q_{n} P_{n l}(\boldsymbol{p}) P_{m j}(\boldsymbol{q}) \\
+\Pi_{i j}(\boldsymbol{k}) q_{l} \Pi_{i m}(\boldsymbol{k}) p_{n} P_{n j}(\boldsymbol{q}) P_{m l}(\boldsymbol{p})
\end{array}\right] .
$$

Again, we use the Markovian approximation to obtain

$$
\begin{aligned}
& \left(\frac{\partial}{\partial t}+v k^{2}\right) E^{d d}(k, t) \\
& =\int_{p+q=k}\left[\int_{-\infty}^{t} \mathrm{e}^{-v\left(k^{2}+p^{2}+q^{2}\right)\left(t-t^{\prime}\right)} \mathrm{d} t^{\prime}\right] \\
& \quad \times \frac{k^{3}}{2 p q} f(k, p, q) E^{s s}(p, t) E^{s s}(q, t) \mathrm{d} p \mathrm{~d} q .
\end{aligned}
$$

Finally, we introduce the eddy damping $\eta^{d}(k)$ and obtain the EDQNM equations for energy spectrum of compressible components:

$$
\begin{aligned}
& \left(\frac{\partial}{\partial t}+v k^{2}\right) E^{d d}(k, t)=\int_{p+q=k} \theta(k, p, q) \\
& \quad \times \frac{k^{3}}{2 p q} f(k, p, q) E^{s s}(p, t) E^{s s}(q, t) \mathrm{d} p \mathrm{~d} q, \\
& \theta(k, p, q) \\
& =\int_{-\infty}^{t} \mathrm{e}^{-\left[v\left(k^{2}+p^{2}+q^{2}\right)+\eta^{d}(k)+\eta^{d}(p)+\eta^{d}(q)\right]\left(t-t^{\prime}\right)} \mathrm{d} t^{\prime} .
\end{aligned}
$$

\section{References}

1. Frisch, U.: Turbulence. The legacy of A. N. Kolmogorov. Cambridge University Press, Cambridge (1995)

2. Sagaut, P., Cambon, C.: Homogeneous Turbulence Dynamics. Cambridge University Press, Cambridge (2008)

3. Bec, J., Khanin, K.: Burgers turbulence. Phys. Rep. 447, 1-66 (2007)

4. Galtier, S., Banerjee, S.: Exact relation for correlation functions in compressible isothermal turbulence. Phys. Rev. Lett. 107, 134501 (2011)

5. Kritsuk, A.G., Wagner, R., Norman, M.L.: Energy cascade and scaling in supersonic isothermal turbulence. J. Fluid Mech. 729, R1 (2013)

6. Falkovich, G., Fouxon, I., Oz, Y.: New relations for correlation functions in Navier-Stokes turbulence. J. Fluid Mech. 644, 465472 (2010)

7. Wagner, R., Falkovich, G., Kritsuk, A.G., et al.: Flux correlations in supersonic isothermal turbulence. J. Fluid Mech. 713, 482-490 (2012)

8. Aluie, H.: Compressible turbulence: the cascade and its locality. Phys. Rev. Lett. 106, 1745902 (2011) 
9. Fauchet, G., Bertoglio, J.P.: A two-point closure for compressible turbulence. C. R. Acad. Sci. Ser. IIB Mech. Phys. Astron. 327, 665-671 (1999)

10. Fauchet, G., Bertoglio, J.P.: Pseudo-sound and acoustic regimes in compressible turbulence. C. R. Acad. Sci. Ser. IIB Mech. Phys. Astron. 327, 673-678 (1999)

11. Bertoglio, J.P., Bataille, F., Marion, J.D.: Two-point closures for weakly compressible turbulence. Phys. Fluids 13, 290-310 (2001)

12. He, G.W., Jin, G.D., Yang, Y.: Space-time correlations and dynamic coupling in turbulent Flows. Annu. Rev. Fluid Mech. 49, 51-70 (2017)

13. Orszag, S.A.: Analytical theories of turbulence. J. Fluid Mech. 41, 363-386 (1970)

14. Leslie, D.C.: Developments in the Theory of Turbulence. Clarendon Press, Oxford (1973)

15. Bos, W.J.T., Bertoglio, J.P.: Lagrangian markovianized field approximation for turbulence. J. Turbul. 14, 99-120 (2013)

16. Kraichnan, R.H.: Kolmogorov hypotheses and Eulerian turbulence theory. Phys. Fluids 7, 1723-1734 (1964)

17. Li, D., Zhang, X., He, G.W.: Temporal decorrelations in compressible isotropic turbulence. Phys. Rev. E 88, 021001(R) (2013)

18. Yang, Y., Pullin, D.I., Bermejo-Moreno, I.: Multi-scale geometric analysis of Lagrangian structures in isotropic turbulence. J. Fluid Mech. 654, 230-277 (2010)

19. Fang, L., Bos, W.J.T., Jin, G.D.: Short-time evolution of Lagrangian velocity gradient correlations in isotropic turbulence. Phys. Fluids 27, 125102 (2015)
20. Chen, J.C., Jin, G.D., Zhang, J.: Large eddy simulation of orientation and rotation of ellipsoidal particles in isotropic turbulent flows. J. Turbul. 17, 308-326 (2016)

21. Sarkar, S., Erlebacher, G., Hussaini, M.Y., et al.: The analysis and modeling of dilatational terms in compressible turbulence. J. Fluid Mech. 227, 473-493 (1991)

22. Li, D., Guo, L., Zhang, X., et al.: A numerical study of a turbulent mixing layer and its generated noise. Sci. China Phys. Mech. 56, 1157-1164 (2013)

23. Porter, D., Pouquet, A., Woodward, P.: Measures of intermittency in driven supersonic flows. Phys. Rev. E 66, 026301 (2002)

24. Kritsuk, A.G., Norman, M.L., Padoan, P., et al.: The statistics of supersonic isothermal turbulence. Astrophys. J. 665, 416-431 (2007)

25. Wang, J.C., Yang, Y.T., Shi, Y.P., et al.: Cascade of kinetic energy in three-dimensional compressible turbulence. Phys. Rev. Lett. 110, 214505 (2013)

26. Bachelor, G.K.: The Theory of Homogeneous Turbulence. Cambridge University Press, Cambridge (1971)

27. Bataille, F., Zhou, Y.: Nature of the energy transfer process in compressible turbulence. Phys. Rev. E 59, 5417-5426 (1999)

28. Pan, L.B., Padoan, P., Kritsuk, A.G.: Dissipative structures in supersonic turbulence. Phys. Rev. Lett. 102, 034501 (2009) 